\title{
Clinical significance of neutrophil gelatinase-associated lipocalin (NGAL) in colorectal cancer: a meta-analysis
}

\author{
Y. Wang ${ }^{1,2}$ and T.T. Zeng ${ }^{3}$ \\ ${ }^{1}$ Laboratory of Molecular Diagnosis of Cancer, West China Hospital, \\ Sichuan University, Chengdu, China \\ ${ }^{2}$ State Key Laboratory of Biotherapy, West China Hospital, \\ Sichuan University, Chengdu, China \\ ${ }^{3}$ Adverse Drug Reaction Monitoring Center of Chengdu, \\ High-Tech District, Chengdu, China \\ Corresponding author: T.T. Zeng \\ E-mail: bettyzeng@foxmail.com
}

Genet. Mol. Res. 13 (3): 7102-7112 (2014)

Received June 4, 2013

Accepted September 20, 2013

Published February 21, 2014

DOI http://dx.doi.org/10.4238/2014.February.21.11

\begin{abstract}
Growing evidence has implicated that neutrophil gelatinase-associated lipocalin (NGAL) plays a role in a spectrum of human cancers. Several observational studies from different parts of the world have been devoted to elucidate the clinical relationship between NGAL and colorectal cancer. This meta-analysis aimed to explore the overall accuracy of NGAL detection for the diagnosis and prognosis of colorectal cancer. Based on comprehensive literature screening on Pubmed, Ovid, and CNKI databases, our screening covered all published papers until March 2013. The relevant papers were selected according to some stringent inclusion criteria. Essential data were extracted from the recruited papers and further processed by systematic meta-analysis. The meta-analysis included 5 studies for diagnosis of colorectal cancer. Overall, the pooled sensitivity and specificity of all studies were $73 \%$ $(95 \% \mathrm{CI}=0.69-0.76)$ and $89 \%(95 \% \mathrm{CI}=0.85-0.93)$. The pooled positive
\end{abstract}


likelihood ratio and negative likelihood ratio were $5.41(95 \% \mathrm{CI}=3.85$ $7.59)$ and $0.37(95 \% \mathrm{CI}=0.22-0.62)$. The pooled diagnostic odds ratios was $18.05(95 \% \mathrm{CI}=11.77-27.69)$. The area under the summary receiver operating characteristic curve for the diagnosis of colorectal cancer was 0.87. Meanwhile, 3 studies were included to evaluate the prognostic significance of NGAL overexpression in colorectal cancer patients. The pooled hazard ratio was $2.12(95 \% \mathrm{CI}=1.35-3.33)$. High level of NGAL predicted poor disease-free survival. Thus, NGAL is a potential biomarker for the diagnosis and prognosis of colorectal cancer.

Key words: NGAL; Colorectal cancer; Diagnosis; Prognosis;

Meta-analysis

\section{INTRODUCTION}

Colorectal cancer is the third most common cause of cancer-related deaths in Western Europe and North America (Bi et al., 2006), and is one of the most common gastrointestinal cancers worldwide (Flower, 1996). Despite extensive research in these 2 decades, the mechanisms underlying colorectal cancer progression and metastasis are still unknown.

If detected at an earlier stage, the majority of colorectal cancer patients can be cured successfully, as there is a strong correlation between the tumor stage of diagnosis and the 5-year survival rate (Davies et al., 2005). With the developments in modern surgery and chemical therapeutics, including molecular-targeted therapy, the 5-year survival rate is still not high, especially for stage IV colorectal cancer patients (Sanjoaquin et al., 2007). The early diagnosis of colorectal cancer is always difficult because of the late onset of symptoms; therefore, many screening tests have been developed, such as fecal occult blood test and colonoscopy. TNM staging is still the traditional technique for prognosis predication in the majority of cases. Therefore, it is important to find an effective tumor biomarker, which can be useful for both diagnosis and prognosis of colorectal cancer.

Neutrophil gelatinase-associated lipocalin (NGAL), also known as lipocalin 2, is a $25-\mathrm{kDa}$ glycoprotein, which was originally identified as a covalent complex with the matrix metalloproteinase-9 in human neutrophils (Triebel et al., 1992). NGAL has been investigated in a variety of physiological and pathological conditions in the recent decade. Primary studies have found that human NGAL is highly homologous to the oncogene products $24 \mathrm{p} 3$ and NRL in mice and rats; thus, it is speculated that NGAL may be a new oncogene in human (Kjeldsen et al., 2000).

In a baseline study conducted by Friedl et al., published in 1999, they comprehensively investigated NGAL expression in human organs and systems and found that colonic adenocarcinomas were strongly NGAL-positive, in contrast to the adjacent normal mucosa. A few studies provided evidence that NGAL acted as a negative prognostic factor for colorectal cancer, as in the case of breast cancer, and even appeared to be a significant predictor of progression in stage I colorectal carcinoma (Barresi et al., 2010). Nevertheless, a study found that NGAL overexpression did not stimulate the growth of colorectal cancer cells either in vitro or in vivo and produced controversial outcomes (Lee et al., 2006).

Therefore, any particular study may not have the persuasiveness for evaluating the 
relationship between NGAL expression and colorectal cancer risk. This study aims to estimate the possibility of NGAL as a biomarker in the diagnosis and prognosis of colorectal cancer via meta-analysis of published literature.

\section{MATERIAL AND METHODS}

\section{Data sources and search strategy}

We followed the Meta-Analysis of Observational Studies in Epidemiology (MOOSE) guidelines for the conduct of meta-analyses of observational cohort studies. Two investigators (Y.W. and T.T.Z.) conducted a literature search on Pubmed, Ovid, and CNKI (China National Knowledge Infrastructure) databases, including all published papers until March 2013, with a combination of the following terms: neutrophil gelatinase-associated lipocalin; NGAL; lipocalin 2; colorectal cancer; colon cancer; and rectal cancer. There were no language restrictions.

\section{Publication selection}

Two reviewers (Y.W. and T.T.Z.) independently reviewed potentially associated publications by checking their titles and abstracts, and then procured the most relevant papers for a further examination. Moreover, the reference lists of the selected papers were screened for any potential information. The criteria used for the literature selection were listed as follows: 1) papers clearly describing studies about the association of NGAL with colorectal cancer; 2) pathological diagnoses and sources of cases and controls should be stated; 3 ) test methods and completeness of data, or any information that may help infer the results should also be offered. Accordingly, the following exclusion criteria were also used: 1 ) design and definition of the experiments were obviously different from those of the selected papers; 2) source of cases and controls and other essential information could not be obtained; and 3) reviews and repeated literature.

\section{Data extraction and study quality assessment}

Two reviewers (Y.W. and T.T.Z.) independently executed data extraction. For conflicting evaluations, an agreement was reached following a discussion. The quality of each study included was assessed using the diagnostic accuracy tool QUADAS (quality assessment for studies of diagnostic accuracy [maximum score 14]). Data including author, publication year, region, study population, the measurement method of NGAL, and completeness of data (the number of true-positive, false-positive, true-negative, and false-negative results to allow reconstruction of the diagnostic 2 by 2 table) were extracted. Meanwhile, to evaluate the prognostic value of NGAL for the survival of colorectal cancer patient, completeness of data (hazard ratios and its confidence intervals, Kaplan Meier curve and its P value, events in each arm) were extracted.

\section{Statistical analysis}

The studies were analyzed using the chi-square-based Q-statistic test to assess het- 
erogeneity and $\mathrm{I}^{2}$ to estimate the degree of heterogeneity. Statistically significant heterogeneity was considered when the P value was less than 0.05 and the $\mathrm{I}^{2}$ value was more than $50 \%$. If there was significant heterogeneity, we used the random-effect model (DerSimonian and Laird). Otherwise, we used the fixed-effect model (MantelHaenszel).

The bivariate model was applied for diagnostic meta-analysis to perform the pooled sensitivity, specificity, positive likelihood ratio (PLR), negative likelihood ratio (NLR), and diagnostic odds ratio (DOR). All the pooled estimates with the corresponding 95\%CI were initially calculated using the appropriate statistical analysis model. We constructed summary receiver operator characteristic (sROC) curves. The area under the curve (AUC) value with $\mathrm{Q}$ value was also calculated to present an overall summary of test performance to differentiate between a diseased and a non-diseased participant. The Spearman correlation coefficient of sensitivity and 1-specificity was calculated to estimate the threshold effect. The publication bias of the studies included was assessed using the effective sample-size funnel plot and the Egger test.

In addition, to evaluate the prognostic significance of NGAL expression in colorectal cancer, the primary endpoint of this analysis was disease-free survival (DFS). The pooled hazard ratios (HR) were estimated according to the Tierney's methods (Tierney et al., 2007) by calculating the 2 following parameters: O-E statistic (the difference between the number of observed and expected events) and log rank variance.

Statistical analysis was implemented by MetaDisc 1.4, Revman 5, and Stata 11.0 softwares.

\section{RESULTS}

\section{Search results and study characteristics}

The systematic literature search generated 28 references based on the search strategy. We excluded 19 studies after screening the titles and abstracts, because they were duplication in these databases, or not relevant to our interests. After a careful review, in the remaining 9 studies, 3 studies were discarded, because of lack of sufficient data for constructing the 2 x 2-contingency tables (Friedl et al., 1999; Conrotto et al., 2008; Zhang et al., 2009b). Because of overlapping of data in the 2 studies (Barresi et al., 2010, 2011), the latest study was chosen (Barresi et al., 2011). Finally, 5 studies were included in this metaanalysis to evaluate the diagnosis value (Nielsen et al., 1996; Chen and Wu, 2007; Zhang et al., 2009a; Barresi et al., 2011; Sun et al., 2011). Among the carefully reviewed 9 studies, 3 studies were recruited to evaluate the prognosis value of NGAL by DFS (Zhang et al., 2009a; Barresi et al., 2011; Sun et al., 2011). A flow chart showing the study selection procedure is given in Figure 1.

We established a database according to the extracted information from these 5 studies. The information was listed in Table 1. Overall, all the selected 5 studies were singlecenter trials from different part of the world and included nearly 857 colorectal cancer patients and health controls. NGAL was measured by IHC in paraffin-embedded sections. The quality of each study was appraised according to QUADAS. The results were shown in Table 1. 


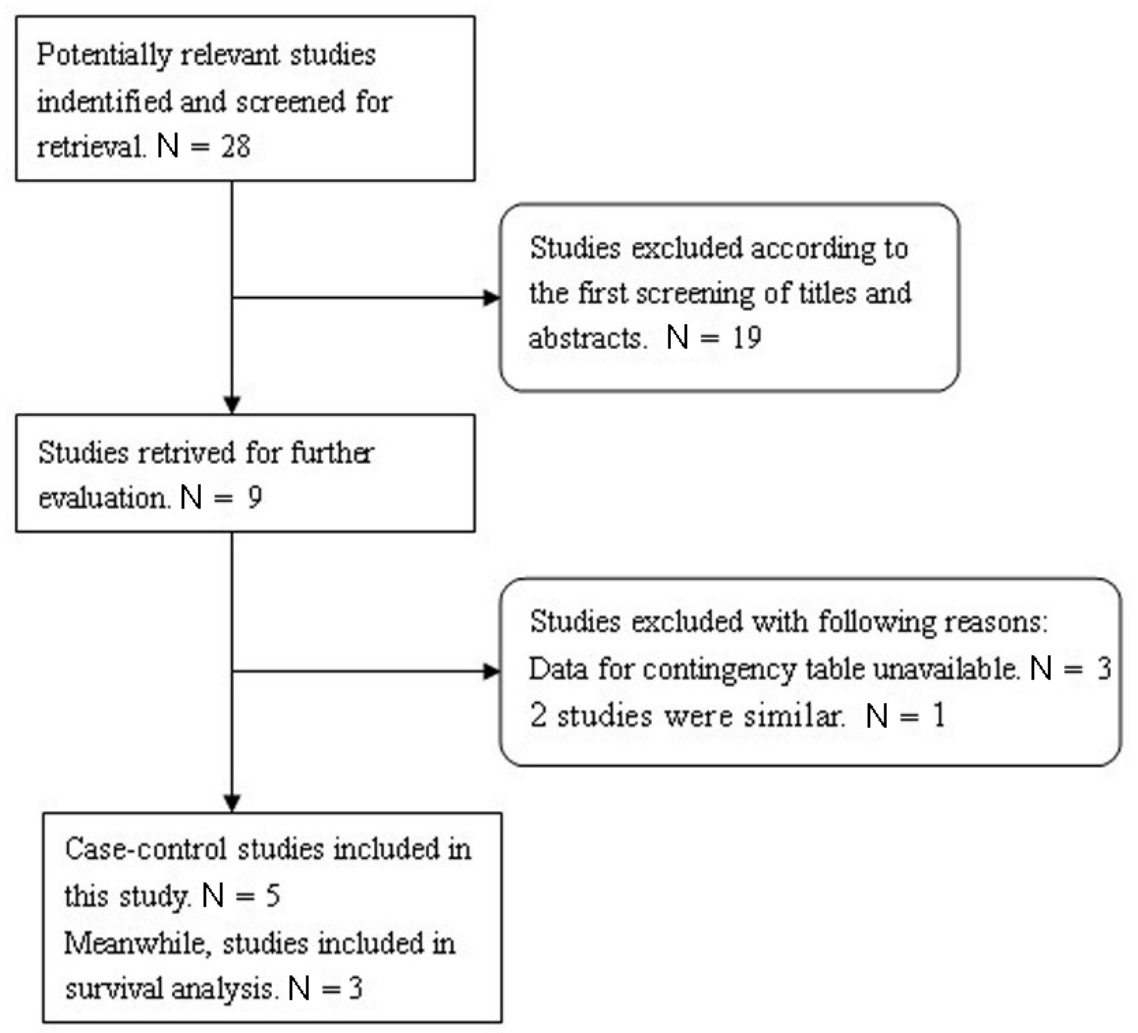

Figure 1. Flow diagram of included/excluded studies.

\begin{tabular}{|c|c|c|c|c|c|c|c|c|}
\hline Author & Year & Method & No. of patients & Type of patients & No. of control & Type of control & Country & QUADAS \\
\hline Zhang et al. & $2009 \mathrm{a}$ & $\mathrm{IHC}$ & 83 & $\begin{array}{l}32 \text { colon cancer, } \\
51 \text { rectum cancer }\end{array}$ & 81 & adjacent normal tissue & China & 11 \\
\hline Chen and Wu & 2007 & $\mathrm{IHC}$ & 23 & colon cancer & 20 & colonic mucosa & China & 12 \\
\hline Sun et al. & 2011 & $\mathrm{IHC}$ & 432 & $\begin{array}{l}287 \text { colorectal cancer, } \\
145 \text { adenoma }\end{array}$ & 94 & normal mucosa & China & 12 \\
\hline Nielsen et al. & 1996 & $\mathrm{IHC}$ & 14 & colorectal cancer & 14 & adjacent normal tissue & Denmark & 13 \\
\hline Barresi et al. & 2011 & $\mathrm{IHC}$ & 48 & colon cancer & 48 & adjacent normal tissue & Italy & 12 \\
\hline
\end{tabular}

$\mathrm{IHC}=$ immunohistochemistry.

\section{Diagnostic accuracy analyses}

The Forest plot of sensitivity, specificity, PLR, NLR, and DOR for the NGAL test in the diagnosis of colorectal cancer was shown in Figures 2 and 3. The overall pooled sensitivity and specificity of all studies were $73 \%(95 \% \mathrm{CI}=0.69-0.76)$ and $89 \%(95 \% \mathrm{CI}=0.85$ $0.93)$, respectively. The overall pooled PLR and NLR were $5.41(95 \% \mathrm{CI}=3.85-7.59)$ and 0.37 $(95 \% \mathrm{CI}=0.22-0.62)$. The pooled DOR was $18.05(95 \% \mathrm{CI}=11.77-27.69)$. 
A

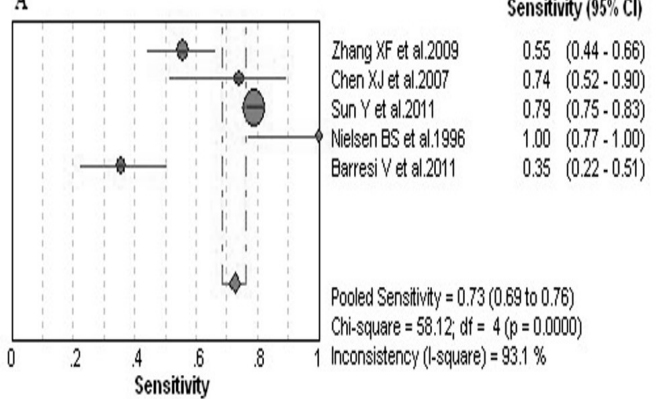

B

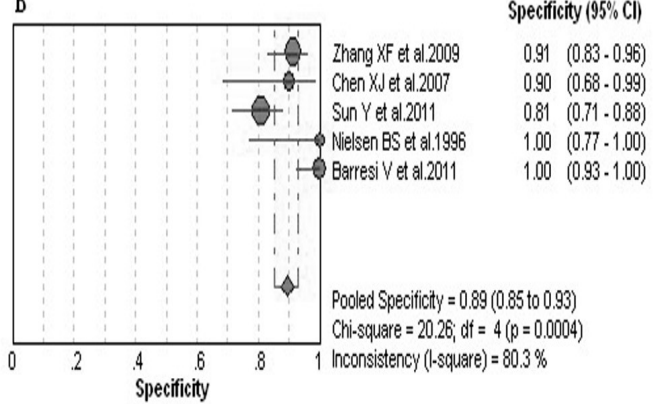

C

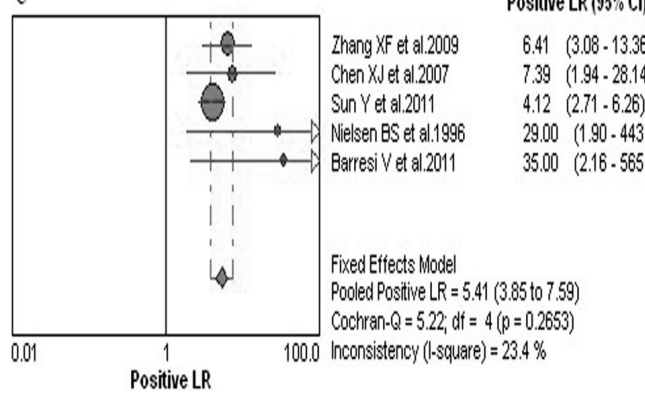

D

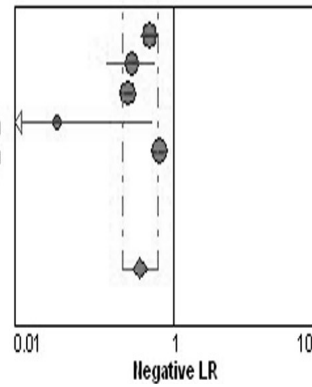

Nlegative LR $(95 \%$ Cl)

$0.49(0.38 \cdot 0.63)$

$0.29(0.14 \cdot 0.59)$

$0.26(0.21-0.32)$

$0.03(0.00 \cdot 0.53)$

$0.65(0.53 \cdot 0.00)$

Random Effects Model

Pooled Negative $L R=0.37(0.22$ to 0.62$)$

Cochran $-Q=45.27$; $d f=4(p=0.0000)$

Inconsistency (.square) $=91.2 \%$

Tau-squared $=0.2465$

Figure 2. Forest plots of the pooled sensitivity (A), specificity (B), positive LR (C), and negative LR (D) of NGAL for the diagnosis of breast cancer. The solid circles represent each individual study and the diamond represents the pooled diagnostic odds ratio. The size of the circle is proportional to the size of the study included. Error bars are $95 \%$ confidence intervals. $\mathrm{LR}=$ likelihood ratio.

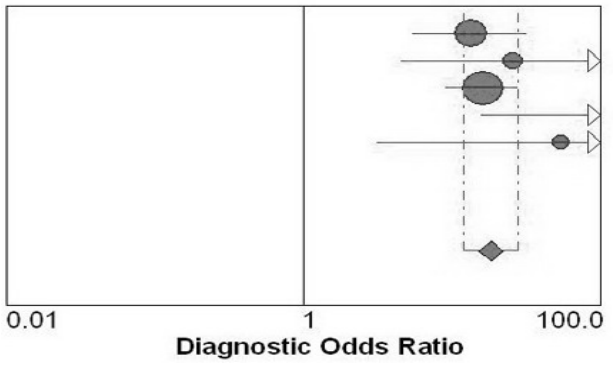

Zhang XF et al.2009 Chen XJ et al. 2007 Sun $Y$ et al. 2011 Nielsen BS et al.1996 Barresi $\vee$ et al.2011

Fixed Effects Model

Pooled Diagnostic Odds Ratio $=18.05$ (11.77 to 27.69 ) Cochran- $Q=4.99 ; \mathrm{df}=4(\mathrm{p}=0.2886)$

Inconsistency $($ I-square $)=19.8 \%$

Figure 3. Forest plots of the pooled diagnostic odds ratio (DOR) of NGAL for the diagnosis of breast cancer. The solid circles represent each individual study and the diamond represents the pooled DOR. The size of the circle is proportional to the size of the study included. Error bars are $95 \%$ confidence intervals.

\section{Summary receiver-operating characteristics}

The sROC curve for NGAL expression showing true-positive rates against false-positive rates from each study displays the trade-off between sensitivity and specificity. The 5 studies were included to construct the sROC curve in Figure 4. The AUC for the diagnosis of colorectal cancer was 0.87 and the $\mathrm{Q}^{*}$ value was 0.8023 . 


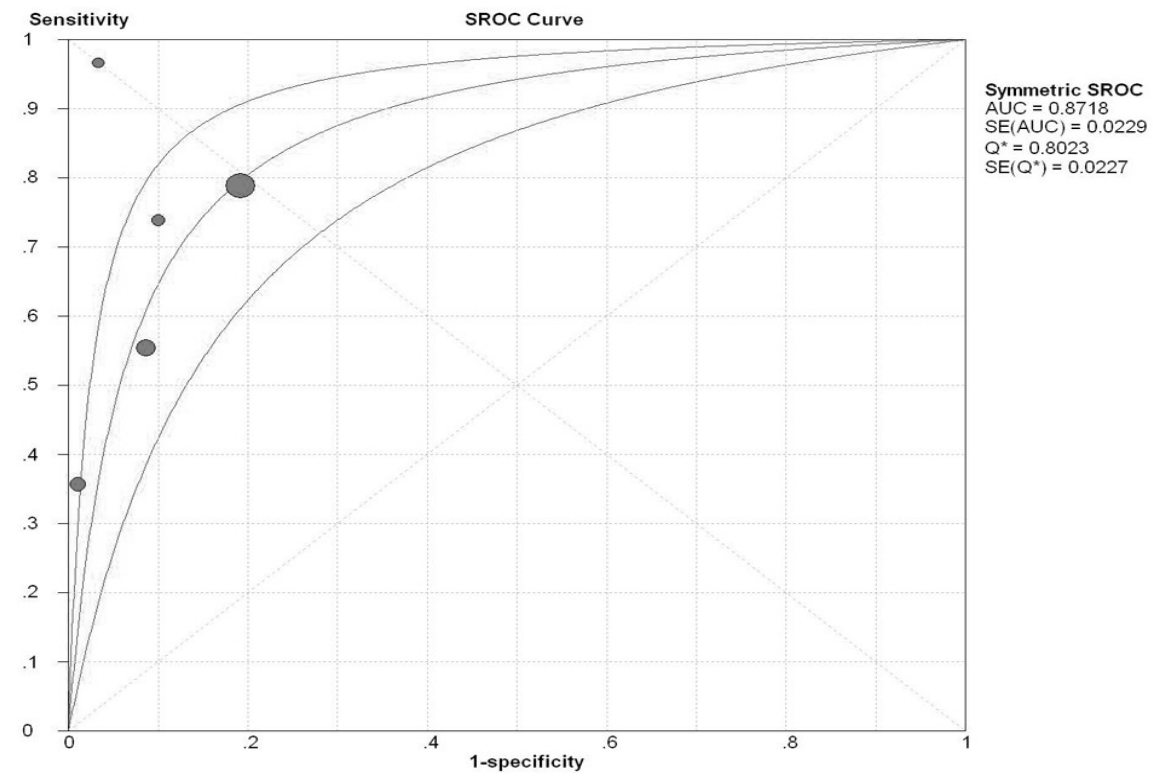

Figure 4. sROC curve of all studies included in the diagnosis of breast cancer. The solid circles represent each individual study in the meta-analysis. The size of the circle is proportional to the size of the study included.

\section{Prognostic value analyses}

The Forest plot of HR for the NGAL test in colorectal cancer prognosis evaluation was shown in Figure 5. The pooled HR was $2.12(95 \% \mathrm{CI}=1.35-3.33)$. High NGAL level in tumor tissue predicted poor DFS.

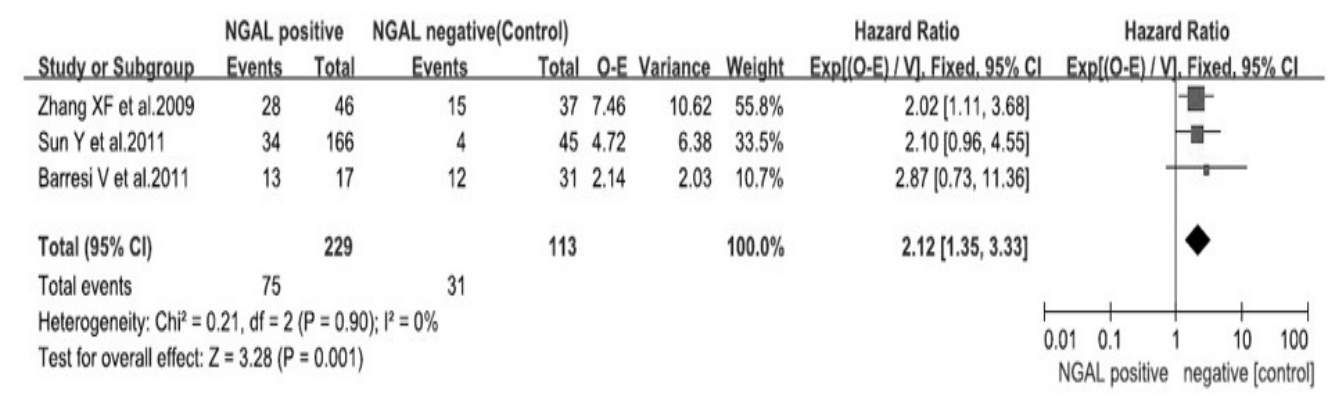

Figure 5. Hazard ratio plot for disease-free survival. The squares represent each individual study and the diamond represents the overall hazard ratio. The size of the square is proportional to the size of the included study. The extremities make the $95 \%$ confidence intervals.

\section{Test of heterogeneity}

We performed a threshold analysis to explore the threshold effect, which was evaluated with the Spearman's correlation coefficient, by using Moses' model weighted by inverse variance. We did not find a statistically significant difference (Spearman's correlation coefficient $=0.4, \mathrm{P}=0.505$ ). 
The Cochran Q-test and the $\mathrm{I}^{2}$ statistic were used to evaluate the presence of statistical heterogeneity occurred in the studies shown in Figures 2, 3, and 5. We found the pooled sensitivity (chi-square $\left.=58.12, \mathrm{I}^{2}=93.1 \%, \mathrm{P}<0.01\right)$, specificity $\left(\right.$ chi-square $=20.26, \mathrm{I}^{2}=$ $80.3 \%, \mathrm{P}<0.05$ ), PLR (chi-square $=5.22, \mathrm{I}^{2}=23.4 \%, \mathrm{P}>0.05$ ), NLR (chi-square $=45.27$, $\left.\mathrm{I}^{2}=91.2 \%, \mathrm{P}<0.01\right), \mathrm{DOR}\left(\right.$ chi-square $\left.=4.99, \mathrm{I}^{2}=19.8 \%, \mathrm{P}>0.05\right)$, and HR (chi-square $=$ $\left.0.21, \mathrm{I}^{2}=0 \%, \mathrm{P}>0.05\right)$.

\section{Publication bias}

Funnel plot was constructed and the Egger test was performed to assess the publication bias of the 5 studies. The shape of funnel plots showed symmetry in Figure 6 . The P value of the Egger test was 0.068 . The result did not suggest any evidence of publication bias.

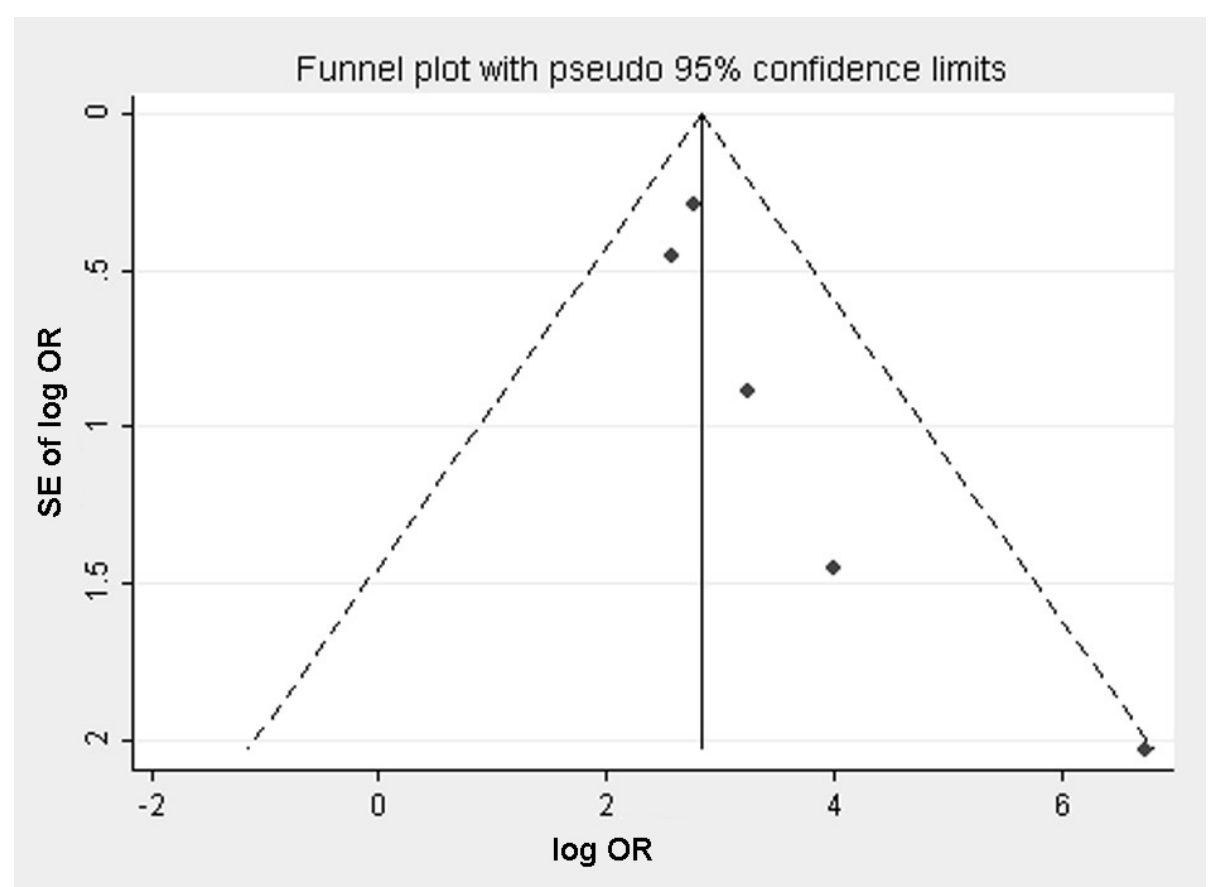

Figure 6. Funnel plot for the assessment of publication bias. Symbols represent each study in the meta-analysis.

\section{DISCUSSION}

NGAL has attracted a rush of scientific investigations in a variety of human cancers worldwide, as a candidate biomarker. In most of the common human cancers, NGAL expression is upregulated, for example in colorectal cancer, gastric cancer (Kubben et al., 2007), esophageal squamous cell carcinoma (Zhang et al., 2007), lung adenocarcinoma (Friedl et al., 1999), primary liver carcinoma (Li et al., 2012), breast cancer (Fernández et al., 2005), 
and thyroid neoplasia (Iannetti et al., 2008), while in a few conditions, NGAL expression is downregulated or remains debatable, for example in pancreatic cancer (Furutani et al., 1998; Laurell et al., 2006; Tong et al., 2008), prostate cancer (Mahadevan et al., 2011), and chronic myeloid leukemia (Villalva et al., 2008).

The mechanisms underlying the cancer-promoting role of NGAL has been explored by some studies, which found that NGAL promoted iron uptake from the extracellular space within the malignant cells to maintain multiplication of neoplastic cells (Devireddy et al., 2005). Elevated NGAL expression in majority of human colorectal carcinoma contributes to colorectal cancer through the alteration of Rac1 cellular distribution and E-cadherin-mediated cell-cell adhesion (Hu et al., 2009).

In this study, we clarified the diagnostic accuracy of NGAL for colorectal cancer by meta-analysis of 5 studies and the results showed the relationship between NGAL and colorectal cancer. Using the bivariate model for diagnostic meta-analysis, we found a summary AUC of 0.87 . The DOR is a single indicator to evaluate the diagnostic value of proposed tests. The pooled DOR of NGAL was 18.05, which indicated the ratio of the odds of positivity in colorectal cancer patients to that in the non-diseased subjects. The pooled sensitivity and specificity were 73 and $89 \%$, respectively, which indicated that the assay might result in $27 \%$ false-negative test results and $11 \%$ false-positive test results. The overall results indicated that NGAL test could be useful in the diagnosis of colorectal cancer. However, the low sensitivity, but high specificity, suggested that a patient with a positive result needed to undergo further laboratory evaluation and imaging.

When PLR $>10$ or NLR $<0.1$, the possibility of approving or negating a diagnosis of a disease significantly increased. In our study, the pooled PLR was 5.41, indicating that the possibility of the NGAL test making a correct diagnosis for positive result is 5.41 times higher than making a wrong diagnosis for positive result. The pooled NLR was 0.37 , indicating that the possibility of the NGAL test making a wrong diagnosis for negative result is 0.37 times higher than making a correct diagnosis for negative result. The overall results indicated that the NGAL protein test had a certain value in the diagnosis of colorectal cancer.

We analyzed the heterogeneity of the 5 studies included. The Spearman's correlation coefficient indicated that the heterogeneity was not related to threshold effect. The funnel plot and the Egger test showed that the meta-analysis did not have publication bias, but the influence of bias on this analysis could not be completely excluded. We may suspect that the heterogeneity was probably due to the small studies with sample sizes.

In this study, we evaluated the prognostic value of NGAL expression in colorectal cancer. When HR $>1$, there is a difference between the 2 groups, and it is a result of causative effect. The pooled HR of $2.12(95 \% \mathrm{CI}=1.35-3.33)$ showed a $112 \%$ relative promotion in the risk of disease progression with high NGAL level. The value of $95 \% \mathrm{CI}>1$ indicated that high NGAL level was a poor influencing factor for the prognosis of colorectal cancer.

Some limitations of the present meta-analysis should be considered. First, studies that met the inclusion criteria were quite few. Some studies reported were excluded because of lack of control groups or lack of certain essential information. Second, IHC was the unique approach for detecting the NGAL in the 5 primary studies included. Third, the sample size of some studies included was small, which did not have adequate power to estimate. In conclusion, because of the limitations of this present meta-analysis, more worldwide studies are required to confirm the value of the NGAL test for the diagnosis or prognosis of colorectal cancer in future.

In summary, the association of NGAL and colorectal cancer was assessed by pooling 
the recruited data via a systematic meta-analysis. The results of the study demonstrated that NGAL might be a potential biomarker for the diagnosis and prognosis of colorectal cancer.

\title{
Conflicts of interest
}

\author{
The authors declare no conflict of interest.
}

\section{REFERENCES}

Barresi V, Luciano R, Vitarelli E, Labate A, et al. (2010). Neutrophil gelatinase-associated lipocalin immunoexpression in colorectal carcinoma: A stage-specific prognostic factor? Oncol. Lett. 1: 1089-1096.

Barresi V, Reggiani-Bonetti L, Di Gregorio C, Vitarelli E, et al. (2011). Neutrophil gelatinase-associated lipocalin (NGAL) and matrix metalloproteinase-9 (MMP-9) prognostic value in stage I colorectal carcinoma. Pathol. Res. Pract. 207: 479-486.

Bi X, Lin Q, Foo TW, Joshi S, et al. (2006). Proteomic analysis of colorectal cancer reveals alterations in metabolic pathways: mechanism of tumorigenesis. Mol. Cell. Proteomics 5: 1119-1130.

Chen XJ and Wu BP (2007). Gene expressions and significance of APC, NGAL, COX-2 in hamartomatous polyps from patient with Peutz-Jeghers syndrome. J. Fourth Mil. Med. Univ. 28: 84-87.

Conrotto P, Roesli C, Rybak J, Kischel P, et al. (2008). Identification of new accessible tumor antigens in human colon cancer by ex vivo protein biotinylation and comparative mass spectrometry analysis. Int. J. Cancer 123: 2856-2864.

Davies RJ, Miller R and Coleman N (2005). Colorectal cancer screening: prospects for molecular stool analysis. Nat. Rev. Cancer 5: 199-209.

Devireddy LR, Gazin C, Zhu X and Green MR (2005). A cell-surface receptor for lipocalin 24p3 selectively mediates apoptosis and iron uptake. Cell 123: 1293-1305.

Fernández CA, Yan L, Louis G, Yang J, et al. (2005). The matrix metalloproteinase-9/neutrophil gelatinase-associated lipocalin complex plays a role in breast tumor growth and is present in the urine of breast cancer patients. Clin. Cancer Res. 11: 5390-5395.

Flower DR (1996). The lipocalin protein family: structure and function. Biochem. J. 318 (Pt 1): 1-14.

Friedl A, Stoesz SP, Buckley P and Gould MN (1999). Neutrophil gelatinase-associated lipocalin in normal and neoplastic human tissues. Cell type-specific pattern of expression. Histochem. J. 31: 433-441.

Furutani M, Arii S, Mizumoto M, Kato M, et al. (1998). Identification of a neutrophil gelatinase-associated lipocalin mRNA in human pancreatic cancers using a modified signal sequence trap method. Cancer Lett. 122: 209-214.

Hu L, Hittelman W, Lu T, Ji P, et al. (2009). NGAL decreases E-cadherin-mediated cell-cell adhesion and increases cell motility and invasion through Rac1 in colon carcinoma cells. Lab. Invest. 89: 531-548.

Iannetti A, Pacifico F, Acquaviva R, Lavorgna A, et al. (2008). The neutrophil gelatinase-associated lipocalin (NGAL), a NF-kappaB-regulated gene, is a survival factor for thyroid neoplastic cells. Proc. Natl. Acad. Sci. U. S. A. 105: 14058-14063.

Kjeldsen L, Cowland JB and Borregaard N (2000). Human neutrophil gelatinase-associated lipocalin and homologous proteins in rat and mouse. Biochim. Biophys Acta 1482: 272-283.

Kubben FJ, Sier CF, Hawinkels LJ, Tschesche H, et al. (2007). Clinical evidence for a protective role of lipocalin-2 against MMP-9 autodegradation and the impact for gastric cancer. Eur. J. Cancer 43: 1869-1876.

Laurell H, Bouisson M, Berthelemy P, Rochaix P, et al. (2006). Identification of biomarkers of human pancreatic adenocarcinomas by expression profiling and validation with gene expression analysis in endoscopic ultrasoundguided fine needle aspiration samples. World J. Gastroenterol. 12: 3344-3351.

Lee HJ, Lee EK, Lee KJ, Hong SW, et al. (2006). Ectopic expression of neutrophil gelatinase-associated lipocalin suppresses the invasion and liver metastasis of colon cancer cells. Int. J. Cancer 118: 2490-2497.

Li LG, Zhou T and Hou YM (2012). Expression and functional analysis of NGAL gene in human hepatocelluar carcinoma. J. Fudan Univ. 51: 91-98.

Mahadevan NR, Rodvold J, Almanza G, Perez AF, et al. (2011). ER stress drives Lipocalin 2 upregulation in prostate cancer cells in an NF-kappaB-dependent manner. BMC Cancer 11: 229.

Nielsen BS, Borregaard N, Bundgaard JR, Timshel S, et al. (1996). Induction of NGAL synthesis in epithelial cells of human colorectal neoplasia and inflammatory bowel diseases. Gut 38: 414-420.

Sanjoaquin MA, Choodari-Oskooei B, Dolbear C, Putcha V, et al. (2007). Colorectal cancer incidence, mortality and 
survival in South-east England between 1972 and 2001. Eur. J. Cancer Prev. 16: 10-16.

Sun Y, Yokoi K, Li H, Gao J, et al. (2011). NGAL expression is elevated in both colorectal adenoma-carcinoma sequence and cancer progression and enhances tumorigenesis in xenograft mouse models. Clin. Cancer Res. 17: 4331-4340.

Tierney JF, Stewart LA, Ghersi D, Burdett S, et al. (2007). Practical methods for incorporating summary time-to-event data into meta-analysis. Trials 8: 16.

Tong Z, Kunnumakkara AB, Wang H, Matsuo Y, et al. (2008). Neutrophil gelatinase-associated lipocalin: a novel suppressor of invasion and angiogenesis in pancreatic cancer. Cancer Res. 68: 6100-6108.

Triebel S, Blaser J, Reinke H and Tschesche H (1992). A $25 \mathrm{kDa}$ alpha 2-microglobulin-related protein is a component of the $125 \mathrm{kDa}$ form of human gelatinase. FEBS Lett. 314: 386-388.

Villalva C, Sorel N, Bonnet ML, Guilhot J, et al. (2008). Neutrophil gelatinase-associated lipocalin expression in chronic myeloid leukemia. Leuk. Lymphoma 49: 984-988.

Zhang H, Xu L, Xiao D, Xie J, et al. (2007). Upregulation of neutrophil gelatinase-associated lipocalin in oesophageal squamous cell carcinoma: significant correlation with cell differentiation and tumour invasion. J. Clin. Pathol. 60: 555-561.

Zhang XF, Zhang XH, Zhou SM and Huang KT, et al. (2009a). NGAL expression in 83 cases with colorectal cancer and its clinical significance. J. Oncol. 15: 115-119.

Zhang XF, Zhang Y, Zhang XH, Zhou SM, et al. (2009b). Clinical significance of Neutrophil gelatinase-associated lipocalin (NGAL) expression in primary rectal cancer. BMC Cancer 9: 134 